Purdue University Purdue e-Pubs

2010

\title{
Pressure and Flow Rate Performance of Piezoelectric Fans
}

\author{
Mark Kimber \\ University of Pittsburgh \\ Kazuhiko Suzuki \\ Sony Corporation \\ Nobutaka Kitsunai \\ Sony Corporation \\ Kenichi Seki \\ Sony Corporation \\ S V. Garimella \\ Purdue University, sureshg@purdue.edu
}

Follow this and additional works at: https://docs.lib.purdue.edu/coolingpubs

Kimber, Mark; Suzuki, Kazuhiko; Kitsunai, Nobutaka; Seki, Kenichi; and Garimella, S V., "Pressure and Flow Rate Performance of Piezoelectric Fans" (2010). CTRC Research Publications. Paper 146.

https://docs.lib.purdue.edu/coolingpubs/146

This document has been made available through Purdue e-Pubs, a service of the Purdue University Libraries. Please contact epubs@purdue.edu for additional information. 


\title{
Pressure and Flow Rate Performance of Piezoelectric Fans
}

\author{
Mark Kimber, Kazuhiko Suzuki, Nobutaka Kitsunai, Kenichi Seki, and Suresh V. Garimella
}

\begin{abstract}
A piezoelectric fan is a flexible cantilever beam whose vibration is actuated by means of a piezoelectric material. Such fans have been employed for the enhancement of heat transfer by increasing the fluid circulation in regions which are otherwise stagnant. The main focus of past studies has been to predict and describe the heat transfer achievable using these devices, as well as the flow field generated by vibrating cantilevers. In order to directly compare these fans with their traditional counterparts such as small axial fans, the present work casts the performance of piezofans in terms of a characteristic often used to represent conventional fans, namely the fan curve. The primary focus of this paper is to determine the relationship between the pressure and the flow rate generated by miniature piezoelectric fans. Experimental measurements are obtained for fans with operating frequencies of 60 and $113 \mathrm{~Hz}$. The maximum flow rate conditions yield nearly $30 \mathrm{l} / \mathrm{min}$, while the greatest static pressure generated is found to be $6 \mathrm{~Pa}$. The performance is highly dependent on both the vibration amplitude and frequency. Predictive relationships are developed to describe the experimental trends and provide insight into the sensitivity of pressure and flow rate to these operating parameters. These fans are directly compared to two commercially available axial fans, both in terms of overall performance and efficiency with which energy is imparted to the fluid. Piezoelectric fans are found to compare quite favorably using either of these performance metrics with a nearly order-of-magnitude increase in fan efficiency. A secondary focus of this paper is to explore the effects of fan installation details on fan performance. The proximity of surrounding walls is considered through the use of three different enclosures within which the fan is mounted. Effective inlet areas from which the air enters the fan are also identified. This paper provides a practical framework for determining the optimal placement and configuration for these fans in prototypical applications.
\end{abstract}

Index Terms-Electronics cooling, fan curves, flow rate, piezoelectric fans, pressure.

\section{NOMENCLATURE}

A Vibration amplitude (mm).

$D \quad$ Piezoelectric fan width (mm).

Manuscript received June 11, 2008; revised October 01, 2008. First published March 24, 2009; current version published November 25, 2009. The work of M. Kimber was supported by the Sony Corporation as part of an internship project conducted in Japan. This work was recommended for publication by Associate Editor F. Shi upon evaluation of the reviewers comments.

M. Kimber was with the Cooling Technologies Research Center, School of Mechanical Engineering and Birck Nanotechnology Center, Purdue University, West Lafayette, IN 47907-2088 USA. He is currently with the Department of Mechanical Engineering and Materials Science, University of Pittsburgh, Pittsburgh, PA 15261 USA.

S. V. Garimella is with the Cooling Technologies Research Center, School of Mechanical Engineering and Birck Nanotechnology Center, Purdue University, West Lafayette, IN 47907-2088 USA (e-mail: sureshg@ @ purdue.edu).

K. Suzuki, N. Kitsunai, and K. Seki are with the Design Technology Solutions Center, Sony Corporation, Mono-Zukuri Technology Center, Tokyo 141-0001, Japan.

Color versions of one or more of the figures in this paper are available online at http://ieeexplore.ieee.org.

Digital Object Identifier 10.1109/TCAPT.2008.2012169 $d \quad$ Distance from fan tip to fan outlet (mm).

$E$ Enclosure within which fan is mounted.

$L_{0} \quad$ Overall piezoelectric fan length (mm).

$L \quad$ Length of exposed portion of fan blade (mm, see Fig. 3).

$P \quad$ Static pressure $(\mathrm{Pa})$.

$P_{\text {in }} \quad$ Power input to fan (mW).

$P_{m} \quad$ Pressure drop across mass flow meter (Pa).

$P_{0} \quad$ Static pressure at zero flow rate condition $(\mathrm{Pa})$.

$Q \quad$ Flow rate (1/min).

$Q_{0} \quad$ Flow rate at zero pressure condition ( $\left.1 / \mathrm{min}\right)$.

Re Reynolds number [see (3)].

$S \quad$ Length of inlet opening (mm, see Fig. 14).

Greek Symbols

$\eta \quad$ Fan efficiency.

$\omega \quad$ Fan driving frequency $(\mathrm{Hz})$.

$\nu \quad$ Kinematic viscosity of air $\left(\mathrm{m}^{2} / \mathrm{s}\right)$.

$\rho \quad$ Density of air $\left(\mathrm{kg} / \mathrm{m}^{3}\right)$.

Subscripts

1,2,3 Enclosure number.

Superscripts

* Dimensionless quantity.

\section{INTRODUCTION}

$\mathbf{P}$ IEZOELECTRIC fans are cantilever beams whose actuation is made possible through a piezoelectric element typically mounted near the cantilever base. As the input signal frequency approaches the fundamental resonance frequency, large vibrations occur at the cantilever tip. This causes motion in the surrounding fluid, which has been shown to provide enhancements in heat transfer with minimal power requirements. Most previous studies have focused on the heat transfer and flow fields generated by these devices, with some of the earliest efforts having been reported by Toda [1], [2] beginning in 1979, who discussed simplified models for the air flow and vibration as well as various possible applications for such devices. Açıkalın et al. [3] addressed the feasibility of their use in products by placing fans in an actual laptop and a simulated cell phone enclosure. They presented experimental results which revealed a considerable increase in heat transfer in both cases. A comparative study between piezoelectric fans, natural convection from a heat sink, and forced convection with axial fans was conducted by Açıalın et al. [4]. They found that piezoelectric fans exhibit 
significant advantages over axial fans by requiring less power; the piezofans were also found to be superior to natural convection heat sinks due to the reduction in volume of the cooling solution. A fundamental study of the heat transfer performance of piezofans, and its dependence on various operating parameters, was conducted by Kimber et al. [5], [6]. The observed behavior was found to share a number of characteristics with impinging jets; correlations were therefore developed based on jet impingement parameters for piezoelectric fans and found to capture the important trends with good accuracy.

These studies, as well as additional investigations [7]-[11], have provided an improved understanding of these novel cooling devices. However, in order to include these fans as a viable option for the thermal engineer, a direct comparison to conventional fans is necessary. Piezofan performance must be cast in terms of common metrics used to describe the behavior of traditional (axial-flow) fans. One of the most often-used fan metrics is the pressure-flow rate relationship, or the fan curve. This is typically used to assess the suitability of fans for a given application. The experimental results in this paper are obtained using standardized techniques [12], so that the performance of piezofans can be evaluated against other traditional or nontraditional fans. Another important contribution of the current study is a determination of the influence of key piezofan parameters (vibration amplitude and frequency) on the overall attainable pressure and flow rate. For conventional fans such as axial fans, guidelines referred to as fan laws [13] suggest how the performance of a certain fan might change with respect to operating variables such as fan diameter and rotation speed. Analogous relationships for piezofans are proposed here, shedding light on the sensitivity of fan performance to various geometric and operational parameters. In the remainder of this paper, the experimental setup is first explained, followed by a description of the parameters under investigation. The experimental results are then presented, followed by a discussion of the effects of fan installation details on fan performance.

\section{EXPERIMENTAL SETUP}

The piezoelectric fans used in this work are commercial products with a piezoelectric actuator bonded to a flexible cantilever beam, or fan blade. The driving frequency is tuned to the first resonance frequency of the fan to provide large oscillations. A typical piezoelectric fan is illustrated in Fig. 1 depicting the important geometric parameters. The footprint of the device is described by the overall length $\left(L_{0}\right)$ and fan width $(D)$. The largest oscillations occur within the portion of the flexible blade not covered by the piezoelectric material. Therefore, the exposed blade length $(L)$ is also of importance in describing the performance and behavior of these devices.

The experimental apparatus used to quantify the flow rate and pressure characteristics consists of an airtight chamber and blower designed according to AMCA standard 210 [12]. This standard outlines different approaches for measuring the pressure and flow rate characteristics for fans of many different types. A schematic diagram of the setup is shown in Fig. 2, in which the fan under investigation is positioned to direct airflow into the chamber. The static pressure generated by the fan $(P)$ is measured with a pressure transducer (Novasina PascalVi-

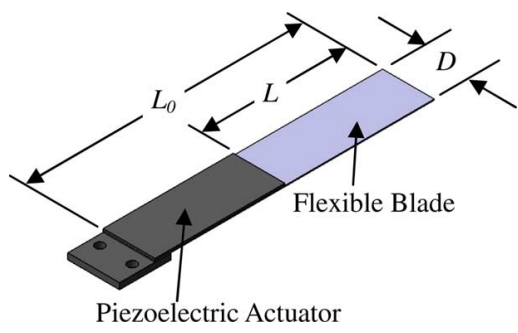

Fig. 1. Illustration of piezoelectric fan showing important geometric parameters: overall blade length $\left(L_{0}\right)$, exposed blade length $(L)$, and fan width $(D)$.

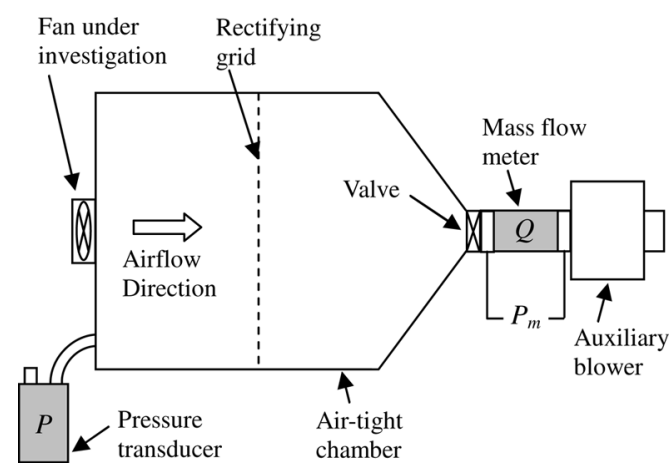

Fig. 2. Illustration of experimental apparatus used to acquire pressure-flow rate measurements.

sion with accuracy $\pm 0.05 \mathrm{~Pa})$. The flow rate $(Q)$ is determined from an additional pressure drop downstream $\left(P_{m}\right)$ through a mass flow meter (accuracy $\pm 11 / \mathrm{min}$ ). An auxiliary blower is employed downstream of the mass flow meter to regulate the flow rate through the system and overcome the pressure drop needed for reliable flow rate measurements.

The measurement procedure begins by closing the valve to capture the attainable pressure at a zero flow rate condition $\left(P_{0}\right)$. Next, the valve is opened and the strength of the auxiliary blower is increased until the static pressure in the chamber $(P)$ reaches zero. This point is the flow rate attainable at a zero pressure condition $\left(Q_{0}\right)$. The strength of the blower is then incrementally decreased yielding nonzero pressure readings in the chamber and flow rates smaller than $Q_{0}$. Each new setting for the blower represents a point along the pressure-flow rate curve which starts at $P_{0}$ and ends at $Q_{0}$. For each experiment conducted in this work, a total of eight points are obtained $\left(P_{0}, Q_{0}\right.$, and 6 intermediate points).

Differences in physical operation of standard axial fans and piezofans dictate the conditions which should be maintained for the piezofan in the experimental apparatus. An axial fan is typically contained within a casing where a certain gap is maintained between the rotating blade and casing. It is clear that the maximum attainable pressure would suffer dramatically if this gap were increased. In order to make a direct comparison between piezoelectric and axial fans, the piezofan assembly is designed to maintain small gaps between the vibrating blade and the casing where the fan is mounted. While a constant radial gap exists for a standard axial fan, casing-to-fan gaps exist in orthogonal directions for piezofans, namely the vibration-parallel (dependent on vibration amplitude) and vibration-orthogonal (dependent only on fan width) directions. This is illustrated 


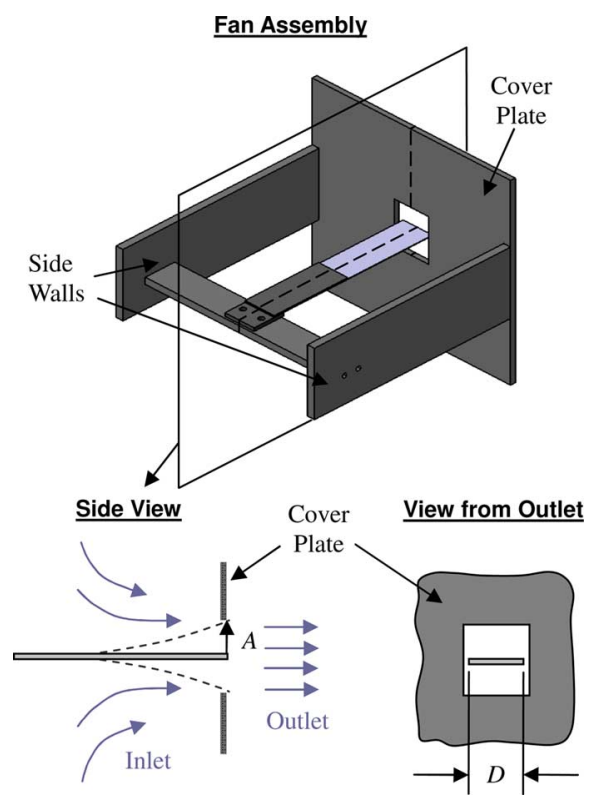

Fig. 3. Illustrations of piezoelectric fan assembly used during experimentation. The outlet dimensions for each case are slightly larger than the fan width $(D)$ and two times the vibration amplitude $(A)$ in the horizontal and vertical dimensions, respectively.

in Fig. 3, where the fan is mounted in an assembly with side walls sufficiently far from the fan (nearly four times the fan width) to remove their possible flow-limiting influence. These walls are attached to a cover plate with a small opening in the center. The side and outlet views shown in Fig. 3 further illustrate the two outlet dimensions of significance. The piezoelectric fans considered in this work have a width of $12.7 \mathrm{~mm}$; therefore, the outlet width for all experiments is set to $15 \mathrm{~mm}$, which leaves a 1.15-mm gap between the vibrating fan and casing in the horizontal direction. The height of the outlet is adjusted to be $1 \mathrm{~mm}$ larger on each side than the vibration amplitude $(A)$ by adjusting the position of thin aluminum strips. These gaps are comparable to those for axial fans of dimensions similar to the piezoelectric fans under investigation. The input voltage is adjusted to obtain the desired vibration amplitude, which is measured using a laser displacement sensor (Keyence LK-G150) to capture the vibration signal of the fan tip. As the amplitude is changed, the aluminum strips are repositioned to maintain the desired vertical gap for all experiments.

The assembly is designed such that the fan tip protrudes $1 \mathrm{~mm}$ beyond the outlet surface. This protrusion distance was initially considered as an additional variable, but the configuration just described is found to provide the greatest pressure and flow rate. This optimum location is somewhat insensitive to protrusion distance within a tolerance of $\pm 2 \mathrm{~mm}$, and is used for all the experimental results presented in this paper.

Similar to an axial fan in the same experimental setup, the piezofan is allowed to pull air from all directions on the suction side. For implementation into an actual device, these conditions will undoubtedly change as inlet obstructions are introduced from the walls of the device. In order to address fan performance and implementation issues separately, the experiments are divided into two sets.

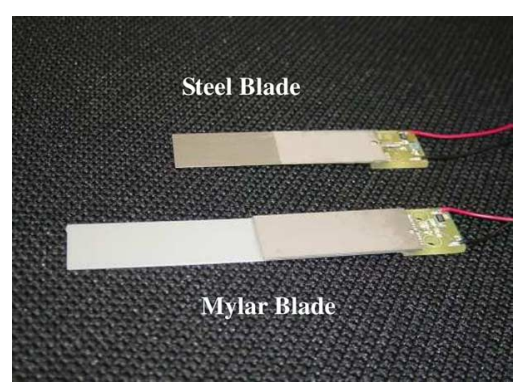

Fig. 4. Two piezoelectric fans (mylar and steel blades) tested to characterize the influence of operating parameters.

TABLE I

LIST OF EXPERIMENTAL AND GEOMETRIC PARAMETERS

\begin{tabular}{lccc}
\hline Parameter & Units & $\begin{array}{c}\text { Mylar } \\
\text { Blade }\end{array}$ & $\begin{array}{c}\text { Steel } \\
\text { Blade }\end{array}$ \\
\hline $\begin{array}{l}\text { Overall } \\
\text { Length }\left(\mathrm{L}_{0}\right)\end{array}$ & {$[\mathrm{mm}]$} & 69 & 47 \\
$\begin{array}{l}\text { Exposed Blade } \\
\text { Length (L) }\end{array}$ & {$[\mathrm{mm}]$} & 36 & 23 \\
Width $(\mathrm{D})$ & {$[\mathrm{mm}]$} & 12.7 & 12.7 \\
Frequency $(\omega)$ & {$[\mathrm{Hz}]$} & 60 & 113 \\
& & $6.0,7.5$, & $3.5,4.5$, \\
Amplitude (A) & {$[\mathrm{mm}]$} & $9.0,10$ & $5.5,6.5$ \\
\hline
\end{tabular}

The first set of experiments employs the fan assembly previously described for the characterization of the pressure and flow rate achieved with vibrating cantilevers. The two fans considered in this first set of experiments have blades made from different materials (mylar and steel) and are shown in Fig. 4. Due to the differences in length and material, the resonance frequency is also different for each fan. The fan dimensions and frequencies are listed in Table I along with the vibration amplitudes considered for each fan. The two fans have identical widths, and therefore, the influence of fan width is not resolved in the current work. The steel fan is shorter, has a higher frequency, and cannot achieve vibration amplitudes that are as high as with the mylar fan. The maximum amplitude for each fan corresponds to the point where the input voltage is slightly below the depoling voltage for the piezoelectric element (approximately 120 and $30 \mathrm{~V}_{\mathrm{rms}}$ for mylar and steel fans, respectively). It is also emphasized that for each experiment, the fans are driven at their first resonance frequency, and the observations from this work are not necessarily applicable to higher resonance frequencies.

The second set of experiments addresses installation effects by mounting the fan in three different enclosures as shown in Fig. 5. The width and height of these enclosures ranges from $40 \times 80 \mathrm{~mm}$ for the largest $\left(E_{1}\right)$ to $15 \times 30 \mathrm{~mm}$ for the smallest $\left(E_{3}\right)$, all with identical lengths of $85 \mathrm{~mm}$. These enclosures consist of four walls: two vertical side walls (aluminum) and two horizontal plexiglass walls above and below the fan. The use of plexiglass allows access for the laser-based vibration measurements. The back side of the enclosure is left open to allow flow from locations upstream of the fan. It should be noted that 


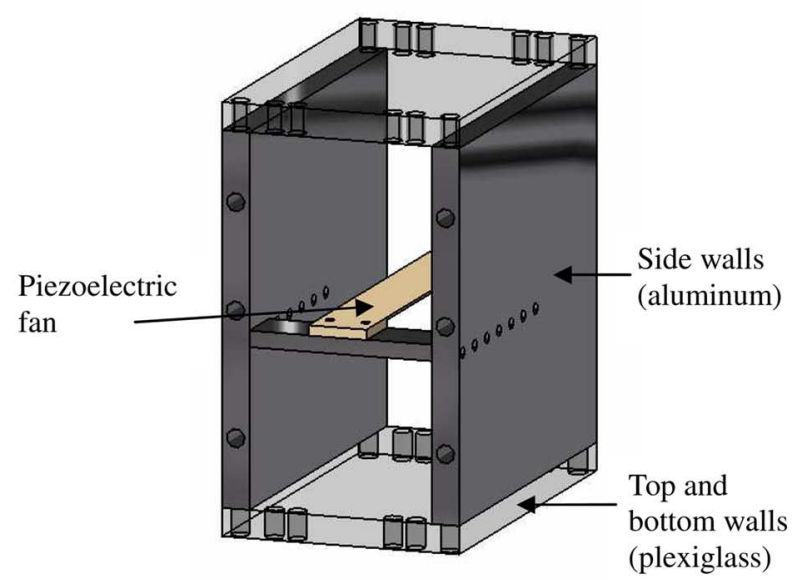

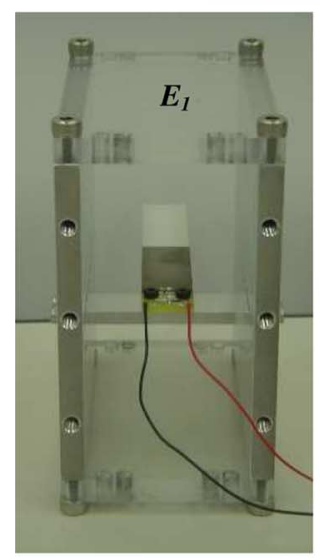

(a)

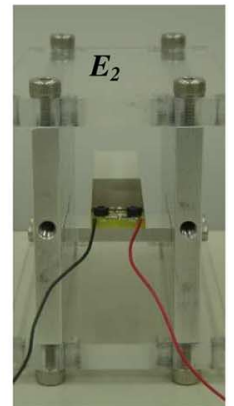

(b)

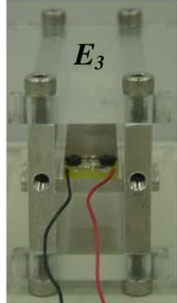

(c)
Fig. 5. Three enclosures $\left(E_{1}, E_{2}, E_{3}\right)$ with cross-sectional dimensions of (a) $40 \times 80 \mathrm{~mm}$, (b) $25 \times 50 \mathrm{~mm}$, and (c) $15 \times 30 \mathrm{~mm}$ used to determine installation effects for piezoelectric fans.

as the distance decreases between the vibrating fan and enclosure walls (from both directions), the fluidic damping on the oscillating blade increases. Because a portion of the power input to the fan is then used in overcoming this additional damping, the power consumption for a given vibration amplitude is substantially greater for $E_{3}$ than for $E_{1}$ (as much as $70 \%$ higher in some cases). For reference, the power needed to drive the fans is dependent on the vibration amplitude and ranges from $10-30 \mathrm{~mW}$ and $20-45 \mathrm{~mW}$ for the mylar and steel fans, respectively. These values represent power consumption with fans mounted in the configuration illustrated in Fig. 3, and remain relatively unchanged when the fans are mounted in $E_{1}$. Any increase in power reported is in reference to these values. Further testing is needed to determine the vibration amplitude as a function of the proximity of surrounding walls for a given power input. All results in this work are presented at a specified amplitude.

The largest source of experimental uncertainty is in the flow rate resolution $( \pm 1 \mathrm{l} / \mathrm{min})$ which results in an uncertainty of less than $10 \%$ in flow rate estimation, while the pressure measurement uncertainties are below 5\% ( \pm 0.05 Pa resolution). Additional uncertainties are noted for vibration amplitude measurements, but are small $(0.05 \%)$ when compared to those of pressure and flow rate.

\section{EXPERIMENTAL RESULTS}

As previously described, the experiments in this work are intended to address two different aspects related to piezofans. The first is to characterize the fan performance given the operating parameters, and the second is to determine the effect of the proximity of enclosure walls to the piezofan. The experimental results are presented in this order.

\section{A. Fan Characterization}

Using the fan assembly illustrated in Fig. 3, the experimentally obtained pressure-flow rate data are shown in Fig. 6 for mylar and steel fans at four different amplitudes each. In all cases, the pressure is highest at the zero flow rate condition (intercept at vertical axis) and then decreases monotonically until the maximum flow rate is reached at the zero pressure condition (intercept at horizontal axis). As expected, the maximum pressure and flow rate both increase with amplitude. The family of curves for either fan type is directly analogous to fan curves for traditional fans at different voltage levels. The main difference is that an increase in voltage for an axial fan causes the rotation speed to increase, whereas for a piezofan, the result is an increase in vibration amplitude. The attainable pressure is a strong function of the fan under investigation, with the steel fan resulting in much higher pressures than the mylar fan. This is true despite the fact that the vibration amplitude is much smaller for the steel fan; however, the frequency is greater for the steel fan by almost a factor of two (see Table I). On the other hand, the maximum flow rate appears to be similar for both fans. Although the mylar fan shows a slight advantage (of roughly $20 \%$ ), the range over the given amplitudes is approximately the same for either fan $(8-28 \mathrm{l} / \mathrm{min})$. The general relationship between pressure and flow rate is readily observed by normalizing each curve with its respective maximum values. This result is shown in Fig. 7, and suggests that the $P-Q$ curve could be estimated with an equation of the form

$$
\left(\frac{P}{P_{0}}\right)=1-\left(\frac{Q}{Q_{0}}\right)^{q}
$$

where $P_{0}$ and $Q_{0}$ are different for each experiment and depend on the operating parameters and fan geometry. For reference, these values are given in Table II for the experiments of interest. With a mean value for the exponent of the flow rate ratio term in (1) equal to 1.6, most of the data is well-described, yielding mean and maximum deviations of 5.6 and $18.9 \%$, respectively.

The performance of piezofans is now compared to that of two small commercially available axial fans in the same experimental setup. These fans were chosen based on their aerodynamic performance such that their maximum values for either flow rate or pressure were in the range of those obtained with the piezoelectric fans. These axial fans are referred to as Axial ${ }_{1}$ and $\mathrm{Axial}_{2}$, representing comparable flow rates and pressure levels, respectively. Data for these two fans are presented in Fig. 8 at two different power inputs each. Also shown for comparison are data from the two largest amplitudes for mylar and steel piezoelectric fans. The maximum attainable pressures for the axial fans are 14.5 and 9.0 Pa for $\mathrm{Axial}_{1}$ and $\mathrm{Axial}_{2}$, respectively, while the corresponding maximum flow rates are 32.6 


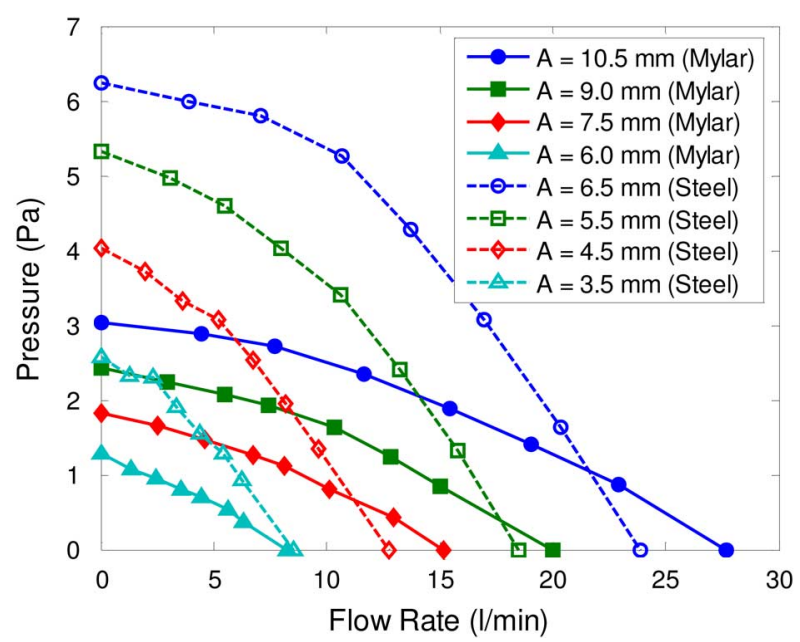

Fig. 6. Experimental pressure-flow rate curves for mylar- and steel-blade piezoelectric fans over a range of vibration amplitudes.

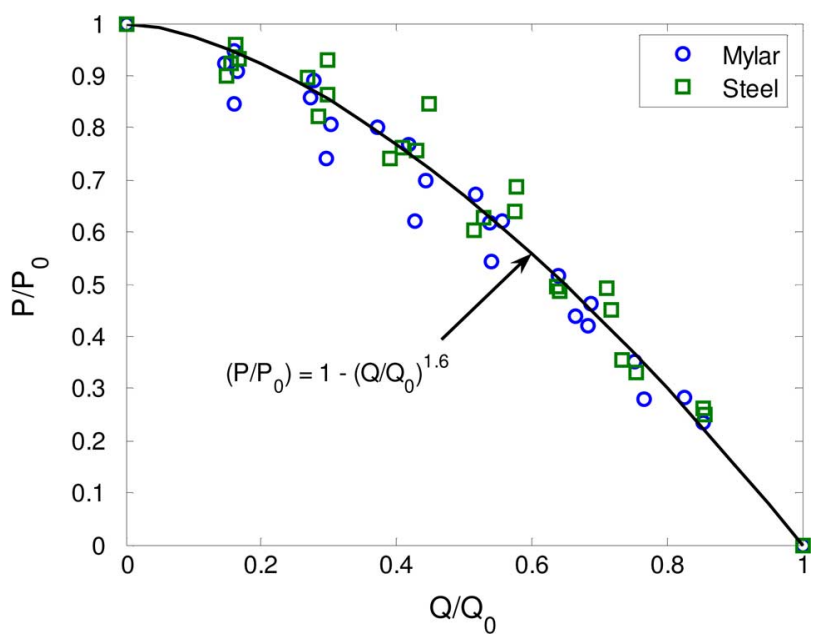

Fig. 7. General $P-Q$ curve represented by (1) obtained from normalizing data in Fig. 6 by $P_{0}$ and $Q_{0}$.

TABLE II

EXPERIMENTAL RESULTS FOR MAXIMUM PRESSURE AND FLOW RATE FOR MYLAR AND STEEL FANS UNDER CONDITIONS ILLUSTRATED IN Fig. 3

\begin{tabular}{lccc}
\hline Fan & $\boldsymbol{A}[\mathrm{mm}]$ & $\boldsymbol{P}_{\boldsymbol{0}}[\mathbf{P a}]$ & $\boldsymbol{Q}_{0}[\mathbf{l} / \mathrm{min}]$ \\
\hline Mylar & 6.0 & 1.28 & 8.28 \\
Mylar & 7.5 & 1.82 & 15.2 \\
Mylar & 9.0 & 2.42 & 20.0 \\
Mylar & 10 & 3.04 & 27.7 \\
Steel & 3.5 & 2.58 & 8.5 \\
Steel & 4.5 & 4.03 & 12.8 \\
Steel & 5.5 & 5.33 & 18.5 \\
Steel & 6.5 & 6.23 & 23.9 \\
\hline
\end{tabular}

and $9.3 \mathrm{l} / \mathrm{min}$. Comparison of the axial fan curves with those for the piezoelectric fans suggests a notable difference in the rate at which pressure drops from its maximum value. A considerably steeper decline in pressure is observed for the axial fans,

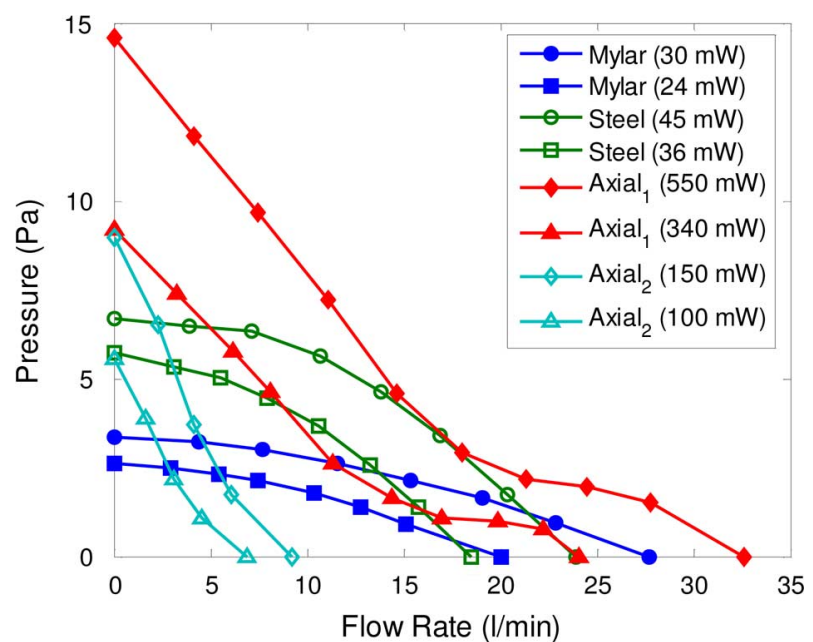

Fig. 8. Experimental fan curves for small axial fans compared to representative results for piezoelectric fans.

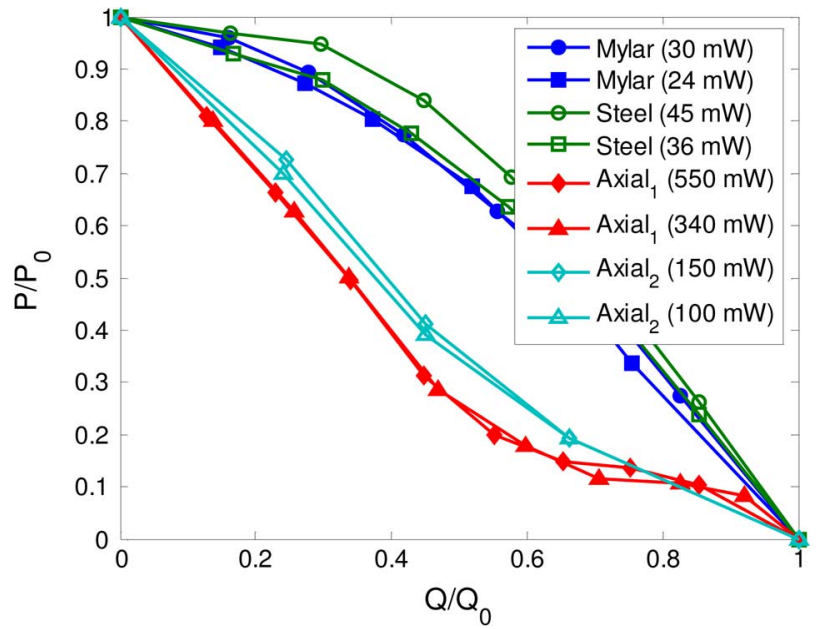

Fig. 9. Fan curves from Fig. 8 normalized by $P_{0}$ and $Q_{0}$.

which can be observed more readily by plotting each fan curve normalized by its maximum pressure and flow rate as shown in Fig. 9. Interestingly, when data from both axial fans are normalized in this fashion, the four curves from the two different axial fans collapse on to a single curve, a behavior which was already shown to exist for piezoelectric fans in Fig. 7. This type of behavior is expected for fans of similar geometry as suggested by well-established fan laws [13]. Analogous relationships for piezoelectric fans are developed in the next section.

Yet another characteristic of the fans that is compared next is the fan efficiency $(\eta)$, which is a measure of the ability of the fan to transform the input power to useful energy imparted to the fluid. Multiple definitions exist for this efficiency resulting in very different values depending on the analysis approach used. Some studies [14], [15] use the ratio of measured pressure to the isentropic pressure, i.e., the pressure attainable through an adiabatic and reversible process. Another approach is to calculate the ratio of the rate of work imparted to the fluid to the overall power input to the fan. This latter definition does not subtract any losses such as those in the electrical to mechanical conversion process, and results in efficiencies that are much lower in 


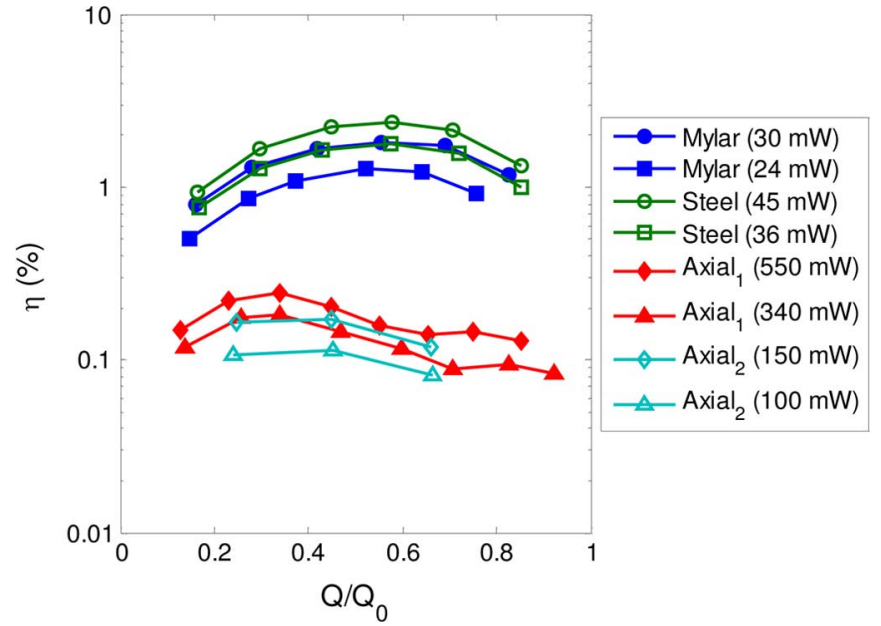

Fig. 10. Fan efficiencies computed for piezoelectric and standard axial fans using (2) and data from Fig. 8.

magnitude. This latter definition is used in this paper because it is a more practical definition. This efficiency is defined as

$$
\eta=\frac{P \cdot Q}{P_{\text {in }}} .
$$

The efficiency is computed at each point along the fan curve for all the data shown in Fig. 8, and presented in Fig. 10 as a function of normalized flow rate. Again, the performance is similar for both the mylar and steel piezoelectric fans, with peak efficiencies obtained in the range of $0.5-0.7$ times the maximum flow rate. Both axial fans also display peak efficiencies that are similar to each other and occur at $0.3-0.5$ times the maximum flow rate. The advantage in efficiency of piezoelectric fans is apparent by comparing the relative magnitude of peak efficiencies, which are 2.4 and $1.8 \%$ for the steel and mylar piezoelectric fans, and 0.25 and $0.18 \%$ for $\mathrm{Axial}_{1}$ and $\mathrm{Axial}_{2}$, respectively. This represents approximately an order-of-magnitude increase in performance in the efficiency of piezofans compared to axial fans, using the efficiency definition in (2).

It is instructive to compare the relative performance of the mylar and steel fans, in terms of the quantities $P_{0}$ and $Q_{0}$ as a function of the variable parameters $A, \omega$, and $L$. Two frequencies, two lengths, and eight total amplitudes are represented in the test matrix. The maximum pressures and flow rates are shown in Fig. 11(a) and (b), respectively, as a function of vibration amplitude. In both graphs, the performance increases with amplitude and the observed trends of variation are similar for both types of fans. As previously mentioned, the range of flow rate seen for the two fans is roughly the same, while the range of attainable pressures is higher for the steel fan. The effect of a change in frequency for a specified vibration amplitude is seen by comparing the largest amplitude for the steel fan $(6.5 \mathrm{~mm})$ and the smallest amplitude for the mylar fan $(6.0 \mathrm{~mm})$. An approximate fourfold increase in pressure and threefold increase in flow rate are observed for the steel fan at this amplitude, suggesting a large dependency on frequency (or alternatively, fan length). In the following section, these results are analyzed further to quantify the influence of fan geometry and operational

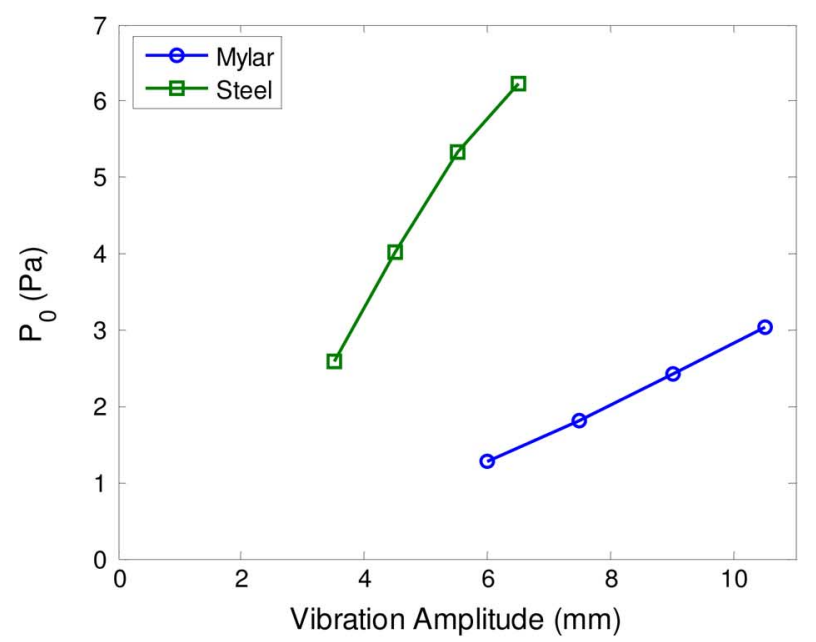

(a)

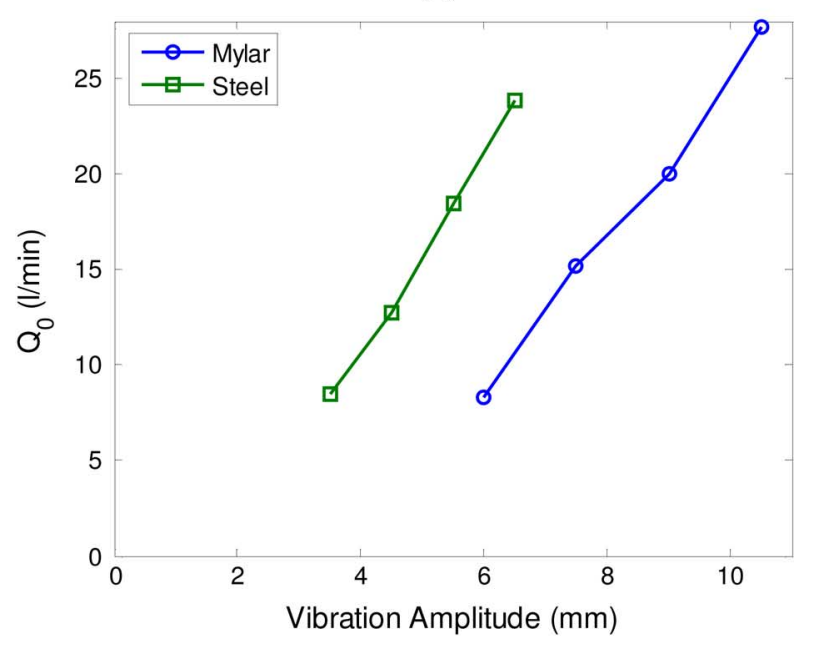

(b)

Fig. 11. Behavior of maximum (a) pressure, and (b) flow rate of the steel and mylar fans with respect to vibration amplitude.

parameters on the performance, and to provide design guidelines.

\section{B. Design Guidelines}

In an effort to generalize the fan performance, appropriate dimensionless pressures and flow rates are defined. Excluding any variation in fluid properties and assuming the beams under investigation are thin (where the thickness of the blade is not significant), one geometrical parameter $(L)$ and two operational parameters $(A, \omega)$ can be utilized to explain the differences seen in the experimental trends for the two fans considered. While the materials from which the two fan blades are made are also different, it can be assumed that the resulting motion in the fluid remains unchanged if all other variables are held constant, since the loading of the fluid is only a function of the motion of the fan. The power needed to drive the fan would, however, depend on the blade material. It should be noted that the operational frequency is also closely tied to the material as well as the length and thickness of the beam (and the piezoelectric actuator properties). 
The Reynolds number (Re) is defined based on the maximum tip velocity $(\omega \mathrm{A})$ and width of the vibrating beam and is expressed as

$$
\operatorname{Re}=\frac{\omega A D}{\nu} .
$$

Dimensionless pressure and flow rate can be obtained using

$$
\begin{aligned}
P_{0}^{*} & =\frac{P_{0}}{\rho \omega^{2} A D} \\
Q_{0}^{*} & =\frac{Q_{0}}{\omega \mathrm{ADL}} .
\end{aligned}
$$

The resulting experimental values for these dimensionless parameters are plotted against Re in Fig. 12. Data from the two different fans collapse to a single curve each for dimensionless pressure and flow rate. In each case, a power-law correlation describes the data with the following equations

$$
\begin{aligned}
& P_{0}^{*}=0.070(\mathrm{Re} / 1000)^{0.561} \\
& Q_{0}^{*}=0.095(\mathrm{Re} / 1000)^{0.888} .
\end{aligned}
$$

Both $P_{0} *$ and $Q_{0} *$ tend to zero as Re approaches zero, i.e., when the frequency or amplitude approaches zero. Using (6) and (7) in conjunction with (3)-(5), it is possible to determine the relative influence of a number of geometric and operating parameters. This gives rise to design guidelines, and suggests the following scaling for maximum pressure and flow rate

$$
\begin{aligned}
P_{0} & \sim \omega^{2.6}, A^{1.6} \\
Q_{0} & \sim \omega^{1.9}, A^{1.9}, L^{0.9} .
\end{aligned}
$$

The form of these guidelines and the manner in which they would be used is similar to fan laws [13] often used for conventional fans to compare the performance of two geometrically similar fans. For piezofans, it is interesting to note that flow rate scales in an identical manner with frequency and amplitude. In general, for cantilevers vibrating in their first resonance mode, a tradeoff exists between these two quantities: as the frequency increases, the attainable amplitude decreases. The current work suggests that these quantities should not be considered in isolation, but rather, it is their product (the tip velocity) which should be maximized in order to obtain the highest flow rate. By this reasoning, if the tip velocity were doubled, a nearly fourfold increase in flow rate would result. For describing the pressure, the frequency has a stronger effect than amplitude. Thus, in situations where increased pressure is desired, the parameter of importance is the frequency rather than the amplitude. For example, an increase of only $10 \%$ in frequency would cause a $28 \%$ increase in pressure, while the same increase in vibration amplitude (10\%) would result in a pressure increase of only $16 \%$. It should be emphasized that the frequency included in (8) and (9) is in reality a function of the geometry and material properties of both the fan blade and piezoelectric material itself [16], [17].

It is emphasized that these guidelines are based upon data from two fans, both of the same width. The influence of the fan width should also be investigated along these lines. It may be expected that when the width is doubled, the flow rate would double as well, but more research is needed with fans of different widths to confirm these expectations.

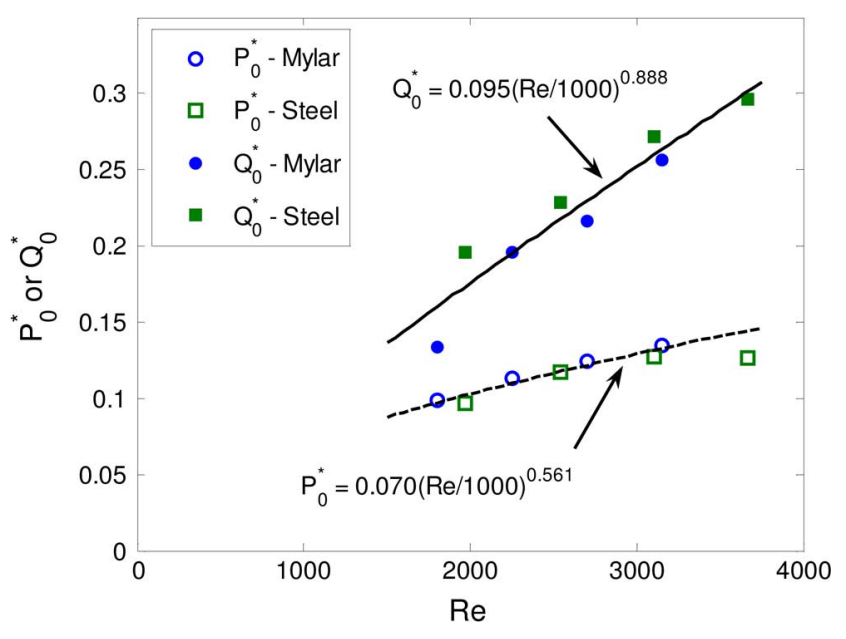

Fig. 12. Maximum dimensionless pressure and flow rate for the two fans as a function of Reynolds number (Re).

\section{Installation Effects}

The factors which should be considered when implementing these devices into actual products are now explored. The effect on the pressure and flow rate as the enclosure around the fan becomes smaller is investigated. It is also important to understand the locations from where the fan draws the inlet flow (i.e., determining the location of the effective "inlet"). Only the mylar fan is considered in this section for the same amplitudes as in Table I. It is assumed that the general trends are consistent for other geometrically similar fans as well.

1) Enclosure Influence: As walls are brought closer to the piezofan from any direction, the power input to the fan needed to maintain a given amplitude increases due to additional viscous drag. As previously mentioned, it is most instructive to present the data in terms of vibration amplitude rather than input voltage as the dependence of amplitude on the proximity of the walls can change with different fans. For the mylar fan mounted within an enclosure, the power requirement increases by $2 \%, 10 \%$, and $40 \%$ for the $E_{1}, E_{2}$, and $E_{3}$ enclosures, respectively (see Fig. 5), when compared to the power needed with no enclosure. Therefore, when the enclosure is sufficiently large, the vibration behavior is relatively unchanged (only $2 \%$ increase in power for $\left.E_{1}\right)$. On the other hand, for the smallest enclosure $\left(E_{3}\right)$, the power increase is large enough that the attainable amplitude is limited by the limitation imposed by the depoling voltage of the piezoelectric element, so that only the smallest two vibration amplitudes ( 6.0 and $7.5 \mathrm{~mm}$ ) can be achieved. Additional experiments are performed using this same enclosure with the plexiglass walls above and below the fan removed. This enclosure configuration is referred to as $E_{3} *$, and is similar in form to the fan assembly illustration shown in Fig. 3, except that the distance between the side walls is now drastically reduced to 15 $\mathrm{mm}$ (the fan width is $12.7 \mathrm{~mm}$ ). This configuration eliminates a large portion of the added drag previously encountered due to the enclosure walls. As a result, the voltages required for the specified amplitudes once again become reasonable.

The most illustrative results are those for the maximum pressure and flow rate, as shown in Fig. 13(a) and (b), respectively. Also included for comparison are data from Fig. 11 for the mylar 
fan with no enclosure. Any deviation in pressure or flow rate from this set of data in either plot signifies the effect of that particular enclosure. For the large enclosure $\left(E_{1}\right)$, the pressure remains relatively unaffected, especially at lower amplitudes. As the amplitude increases, the curve for $E_{1}$ starts to deviate, suggesting that the size for the enclosure to remain nonintrusive must be bigger for larger amplitudes. Pressures with enclosure $E_{2}$ reveal the adverse effect of confinement, which is again more pronounced as the amplitude is increased. At the largest amplitude $(10.5 \mathrm{~mm})$ in $E_{2}$ the attainable pressure is reduced by nearly a factor of two. Enclosure $E_{3}$ behaves very similarly to $E_{2}$, suggesting that the reduction in pressure has leveled off. Only the smallest two amplitudes could be tested in $E_{3}$. The most intriguing behavior occurs for the $E_{3} *$ configuration where the walls above and below the fan are removed. The pressure has now increased substantially, providing a 30\% advantage over the case without an enclosure. For an axial fan, greater pressures at the same rotational speed imply a tighter pressure seal. A similar conclusion may be drawn regarding the piezofans here, suggesting that with sidewalls so close to the vibrating fan and top and bottom walls removed, the result is a tighter pressure seal.

The flow rate is less sensitive to surrounding walls as seen in Fig. 13(b), only showing a deviation for the smallest enclosure. However, in the case of $E_{3} *$, the flow rate again matches the other cases. This suggests that the attainable flow rate is indeed a function only of the operating and geometric parameters of the fan. It is argued that enclosure $E_{3}$ limits the flow by removing some of the inlet area. This effect is treated in more detail in the next section, but implies that the flow primarily enters from above and below the vibrating fan as opposed to lateral or upstream locations of the fan. Therefore, the proximity of the side walls has little impact on the attainable flow rate. An experiment where walls slowly approach the vibrating fan from above and below would be analogous to experiments often conducted with axial fans where a flat plate placed upstream of the fan slowly approaches the inlet [18]. Just as in the case of axial fans, it is expected that there is a certain distance below which the flow rate begins to be affected and experiences a sharp drop.

2) Inlet Considerations: With the intention of exploring significant parameters with respect to the inlet side of the fan, additional experiments are performed using a slightly modified $E_{3} *$ configuration. From the previous section, it is suggested that the "effective inlet" location for a piezofan can be considered to be directly above and below the vibrating cantilever. The two different configurations used for these inlet experiments are shown in Fig. 14, where the length of the inlet opening $(S)$ is measured from the fan tip and represents the portion of the top and bottom walls which are removed. Air can be pulled through this opening as well as from upstream of the fan for the configuration in Fig. 14(a). For the second configuration [Fig. 14(b)], the upstream side of the fan is sealed off, thereby allowing airflow through only the top and bottom openings. The first and second configurations found in Fig. 14(a) and (b) are referred to as inlet ${ }_{A}$ and inlet ${ }_{B}$, respectively. A vibration amplitude of 9 $\mathrm{mm}$ is maintained for these experiments.

Results from these experiments are shown in Fig. 15(a) and (b) for the maximum pressure and flow rate, respectively. For the largest inlet opening $(S=32 \mathrm{~mm})$, both the pressure and

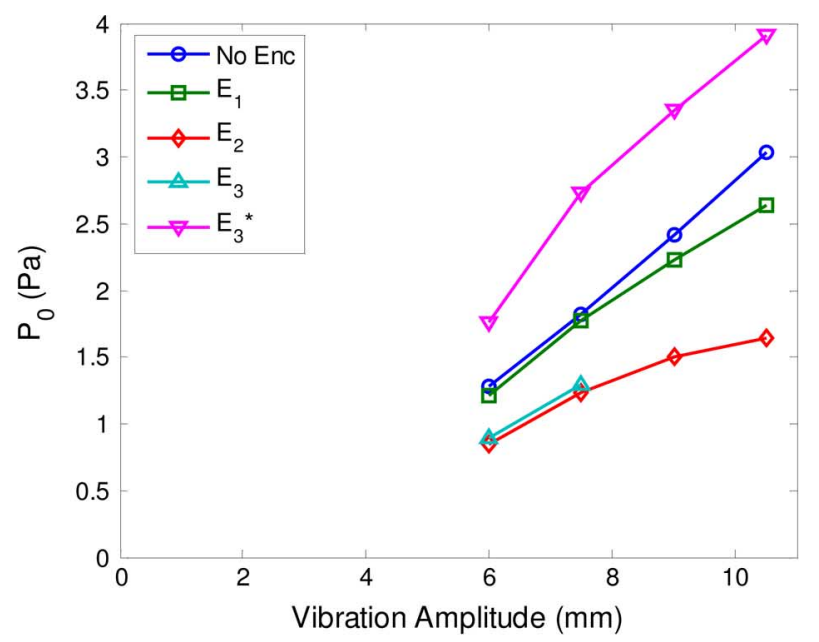

(a)

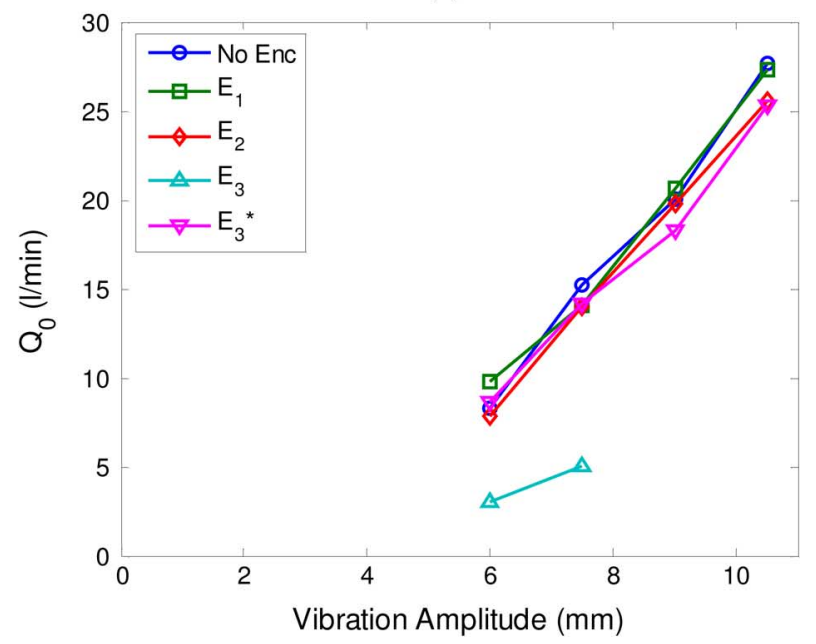

(b)

Fig. 13. Effect of various enclosure conditions for maximum (a) pressure, and (b) flow rate. $E_{3} *$ refers to the condition with the fan mounted in enclosure $E_{3}$ with the top and bottom walls removed.

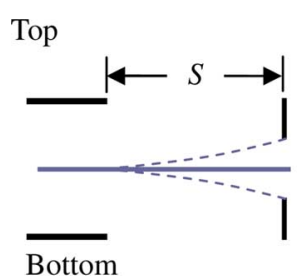

(a)

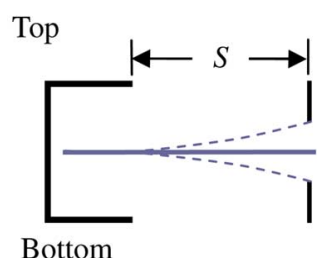

(b)
Fig. 14. Illustration of opening $(S)$ for inlet experiments where (a) top and bottom walls are partially removed, and (b) same as (a) with upstream blocked off in addition.

flow rate are approximately equal to values obtained from the $E_{3}^{*}$ configuration. The impact of the upstream blockage is minimal as well, suggesting that nearly all the flow for this inlet size is drawn from above and below the vibrating fan. It is important to note that the size of the inlet in this case is nearly equal to the exposed blade length of the mylar fan under investigation (see Table I). It appears that if the top and bottom walls do not cover blade locations experiencing large vibration amplitudes, the performance remains relatively unchanged. As the inlet opening 


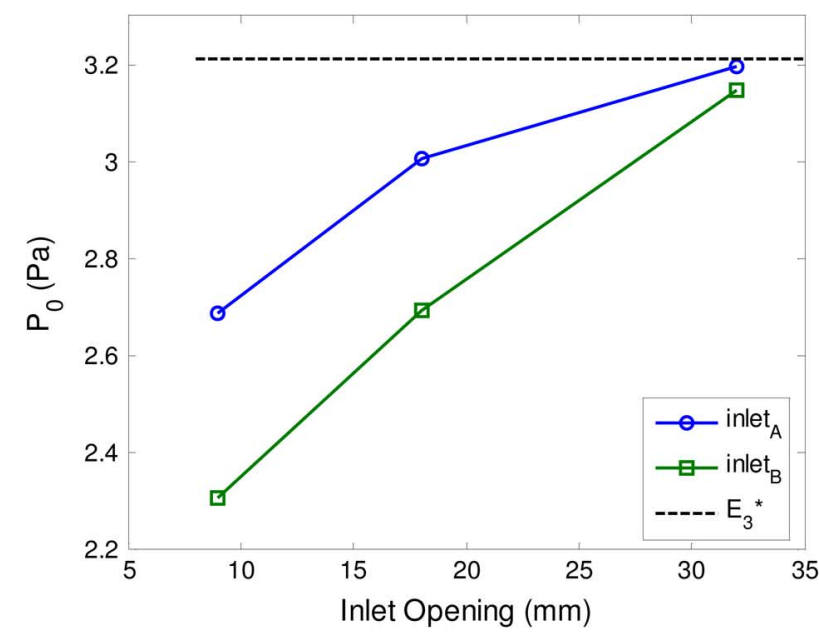

(a)

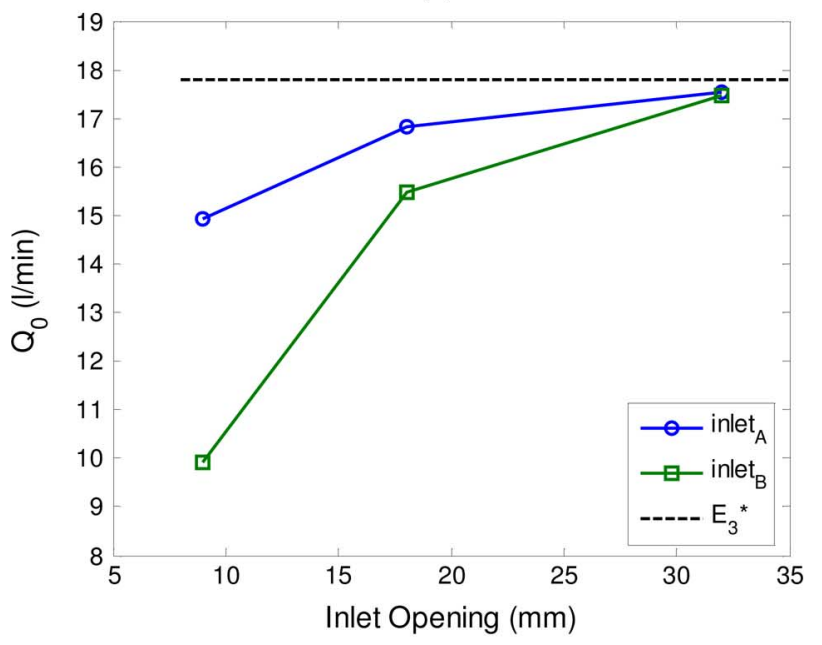

(b)

Fig. 15. Effect of inlet opening on maximum (a) pressure, and (b) flow rate for piezofan placed in $E_{3}$ with different openings $S$ and with and without an upstream blockage.

becomes smaller, the top and bottom walls begin to cover more of the exposed blade. The adverse effect of this is observed in Fig. 15 in both the attainable pressures and flow rates. The effect of upstream blockage also has a larger influence when the inlet is small. For example, for the smallest inlet opening $(S=9$ $\mathrm{mm})$, a $35 \%$ decrease in flow rate occurs in the presence of the upstream blockage relative to the no-blockage case. A general guideline that emerges is that any top and bottom walls should not be placed near portions of the fan which experience large vibrations. If this is not an option for a particular application, such walls should be positioned sufficiently far from the fan, at a distance of 4-5 vibration amplitudes for the flow rate to remain unchanged, and slightly farther (6-7 vibration amplitudes) for the pressure to remain unchanged.

\section{CONCLUSION}

Experimental measurements of the pressure and flow rate characteristics of piezoelectric fans are presented. This work enables a direct comparison of these devices to other fans for which fan curves are available. Preliminary design guidelines are developed from which the relative influence of a number of important parameters can be determined.

The key findings regarding the aerodynamic performance of piezofans are as follows.

1) Compared to standard axial fans with similar pressure or flow rate levels, piezoelectric fans are nearly 10 times more efficient in converting the input power to useful energy imparted to the fluid.

2) The attainable flow rate exhibits a nearly quadratic dependence on the tip velocity.

3) The vibration frequency is more influential in determining the attainable pressure compared to the vibration amplitude.

The installation effects can be summarized as follows.

1) A large enclosure $\left(E_{1}\right)$ has relatively little influence on the pressure or flow rate.

2) As the size of the enclosure becomes somewhat smaller $\left(E_{2}\right)$, only pressure is adversely affected.

3) There is a certain enclosure size (between $E_{2}$ and $E_{3}$ ) where the flow rate begins to be affected and a sharp drop in performance is observed.

In addition, the inlet flow is found to be drawn primarily from above and below the portions of the vibrating fan experiencing the largest amplitude, and these portions should remain uncovered to ensure the largest flow rate possible. Thermal performance of such fans has been investigated in past studies [3]-[7], [10] and is not considered here. It is recommended that the thermal performance of piezoelectric fans and axial fans be compared under conditions of identical pressure and flow rate characteristics.

\section{REFERENCES}

[1] M. Toda, "Theory of flow generation by a resonant type PVF2 bimorph cantilever vibrator," Ferroelectrics, vol. 22, pp. 911-918, 1979.

[2] M. Toda, "Voltage-induced large amplitude bending device-PVF2 bimorph-Its properties and applications," Ferroelectrics, vol. 32, pp. 127-133, 1981.

[3] T. Açıkalın, S. M. Wait, S. V. Garimella, and A. Raman, "Experimental investigation of the thermal performance of piezoelectric fans," Heat Transfer Eng., vol. 25, no. 1, pp. 4-14, 2004.

[4] T. Açıkalın, I. Sauciuc, and S. V. Garimella, "Piezoelectric actuators for low-form factor electronics cooling," in Proc. Summer Heat Transfer Conf., 2005, vol. 2, pp. 939-943.

[5] M. Kimber, S. V. Garimella, and A. Raman, "Local heat transfer coefficients induced by piezoelectrically actuated vibrating cantilevers," ASME J Heat Transfer, vol. 129, pp. 1168-1176, 2007.

[6] M. Kimber and S. V. Garimella, "Local heat transfer coefficients under flows induced by vibrating cantilevers," ASME J. Heat Transfer, vol. 129, no. 8, p. 933.

[7] T. Açıkalın, S. V. Garimella, A. Raman, and J. Petroski, "Characterization and optimization of the thermal performance of miniature piezoelectric fans," Int. J. Heat Fluid Flow, vol. 28, no. 4, pp. 806-820, 2007.

[8] Y. Kim, S. T. Wereley, and C. Chun, "Phase-resolved flow field produced by a vibrating cantilever plate between two endplates," Phys. Fluids, vol. 16, no. 1, pp. 145-162, 2004.

[9] J. Petroski, M. Arik, and M. Gursoy, "Piezoelectric fans: Heat transfer enhancements for electronics cooling," in Proc. Summer Heat Transfer Conf., HT2008-56045, 2008, pp. 1-7.

[10] S. M. Wait, S. Basak, S. V. Garimella, and A. Raman, "Piezoelectric fans using higher flexural modes for electronics cooling applications," IEEE Trans. Compon. Packag. Technol., vol. 30, no. 1, pp. 119-128, Mar. 2007.

[11] T. Açıkalın, A. Raman, and S. V. Garimella, "Two-dimensional streaming flows induced by resonating, thin beams," J. Acoust. Soc. Amer., vol. 114, no. 4, pp. 1785-1795, 2003.

[12] Laboratory Methods of Testing Fans for Certified Aerodynamic Performance Rating, ANSI/AMCA Standard 210-07. 
[13] R. Jorgensen, Fan Engineering. Buffalo, NY: Buffalo Forge Co., 1970.

[14] R. Grimes, E. Walsh, D. Quin, and M. Davies, "Effect of geometric scaling on aerodynamic performance," AIAA $J$, vol. 43, no. 11, pp. 2293-2298, 2005.

[15] R. A. Wallis, Axial Flow Fans and Ducts. New York: Wiley, 1983.

[16] S. Basak, A. Raman, and S. V. Garimella, "Dynamic response optimization of piezoelectrically excited thin resonant beams," J. Vibr. Acoust., vol. 127, pp. 18-27, 2005.

[17] P. Burmann, A. Raman, and S. V. Garimella, "Dynamics and topology optimatization of piezoelectric fans," IEEE Trans. Compon. Packag. Technol., vol. 25, no. 4, pp. 592-600, Dec. 2003.

[18] S. C. Lin and C. A. Chou, "Blockage effect of axial-flow fans applied on heat sink assembly," Appl. Thermal Eng., vol. 24, pp. 2375-2389, 2004.

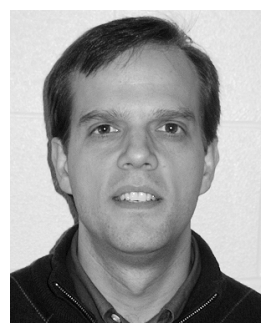

Mark Kimber received the B.S.M.E. and M.S.M.E. degrees from Brigham Young University, Provo, UT, in 2002 and 2004, respectively, and the Ph.D. degree in mechanical engineering in 2008 from Purdue University, West Lafayette, IN.

$\mathrm{He}$ conducted thermal and fluidic studies of piezoelectric fans for use as low-power heat transfer enhancement devices. His current research interests as an Assistant Professor at the University of Pittsburgh include energy accountability and sustainability in electronic equipment, energy efficient and biomimetic methods of propulsion, and innovative heat transfer methods pertaining to nuclear energy generation.

Dr. Kimber was the recipient of the Laura Winkelman Davidson Fellowship (2006-2007) and the Graduate Student of the Year Award in the School of Mechanical Engineering (2008)

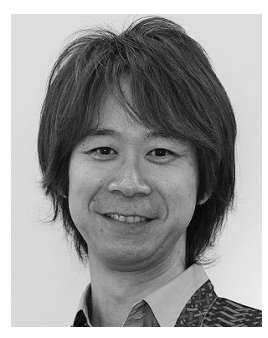

Kazuhiko Suzuki received the B.Eng. and M.Eng. degrees in fluid mechanics from Tokyo University of Science, Tokyo, Japan, in 1996 and 1998, respectively.

Upon graduation he has worked at Sony Corporation and developed surface-mount technology for broadcast products for five years. Currently, he analyzes heat transfer designs of the thermal management solutions for varied consumer products. He also engages in research and development for thermal devices.

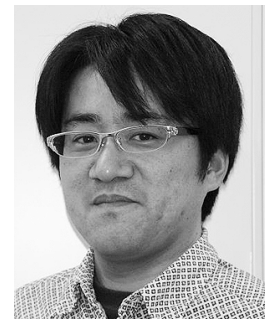

Nobutaka Kitsunai received the B.S. and M.S. degrees in mechanical system engineering from Tokyo University of Agriculture and Technology, Tokyo, Japan, in 1998 and 2000, respectively.

$\mathrm{He}$ is currently with Sony Corporation, Tokyo, working in the area of thermal analysis and thermal design of chip packaging, chassis, and system-level packaging.

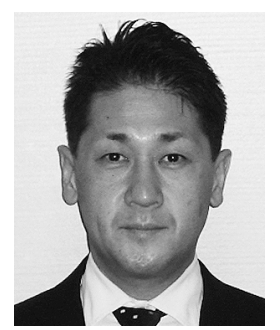

Kenichi Seki, received the B.S. degree from Sophia University, Tokyo, Japan, in 1987. He is currently pursuing the Ph.D. degree in the Graduate School of System Design and Management, Keio University, Tokyo, under the supervision of Prof. Nishimura.

$\mathrm{He}$ is Deputy General Manager and Distinguished Engineer of the Mono-Zukuri Group, Sony Corporation, Tokyo. His research area includes design methodology development for thermal and acoustic problems of consumer products.

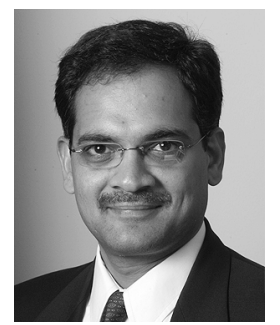

Suresh Garimella received the Ph.D. degree from the University of California at Berkeley in 1989.

$\mathrm{He}$ is the R. Eugene and Susie E. Goodson Professor of Mechanical Engineering at Purdue University, West Lafayette, IN. He is Director of the NSF Cooling Technologies Research Center, the Electronics Cooling Laboratory, and the Solidification Heat Transfer Laboratory. His research interests include thermal microsystems, high-performance compact cooling technologies, electrothermal codesign and electronics packaging, micro- and nano-scale transport phenomena, and materials processing. He has worked with over 60 graduate students and 20 visiting scholars and postdoctoral researchers, and has coauthored over 300 refereed journal and conference publications, besides editing or contributing to a number of books. He serves as an Associate Editor for ASME Thermal Science and Engineering, and as an Editor of Applied Energy and Experimental Heat Transfer, and has served as an Associate Editor for the ASME Journal of Heat Transfer and an Editor of Heat Transfer-Recent Contents, and on the Editorial Board of Experimental Thermal and Fluid Science.

Dr. Garimella is a Fellow of the ASME. His efforts in research and engineering education have been recognized with the 2004 ASME Gustus L. Larson Memorial Award, K16 Clock Award from the ASME, Graduate School/UWM Foundation Research Award in recognition of Outstanding Research and Creative Activity (1995), UWM Distinguished Teaching Award in recognition of Demonstrated Dedication to Excellence in Undergraduate Instruction (1997), and Society of Automotive Engineers' Ralph R. Teetor Educational Award (1992). 